\title{
A Call to Action to Implement Effective COVID-19 Prevention and Screening of Individuals with Severe Intellectual Developmental and Autism Spectrum Disorders
}

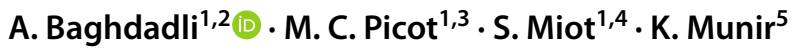 \\ Published online: 30 September 2020 \\ ○) Springer Science+Business Media, LLC, part of Springer Nature 2020
}

The Coronavirus Disease 2019 (COVID-19) is an unprecedented pandemic with major life-threatening complications among people with serious underlying conditions, especially older adults. Today, in France, COVID-19 screening tests are performed on symptomatic individuals, inpatients, healthcare and emergency workers, but many individuals are asymptomatic and carry the virus. Screening is currently established in hospitals or clinical laboratories by means of reverse transcription polymerase chain reaction (RT-PCR) tests that utilize deep nasopharyngeal swabs, with possibility of false negatives due to insufficient amount of viral material collection or laboratory error. Rapid serological diagnosis could be deployed soon to test larger set of subjects. The challenge is to define optimal screening strategies to identify both asymptomatic subjects and subgroups at risk of severe complications.

Since the fatality rate has been rising steeply in longterm care facilities for the elderly, screening tests have been scaled up to triage care of infected subjects in these settings. Seminal research supports the need to systematize these efforts and to better understand the type of care that

A. Baghdadli

rech-clinique-autisme@chu-montpellier.fr

1 Center of Excellence in Autism and Neurodevelopmental Disorders, University and CHU of Montpellier, Montpellier, France

2 Center of Resources in Autism, University and CHU of Montpellier, 39 Avenue Charles Flahaut, 34295 Montpellier Cedex 05, France

3 Clinical Research and Epidemiology Unit, University and CHU of Montpellier, 34000 Montpellier, France

4 Geriatrics Department, Center of Excellence in Autism and Neurodevelopmental Disorders, University and CHU of Montpellier, 34000 Montpellier, France

5 Developmental Medicine Center, Boston Children's Hospital, Harvard Medical School, Boston, MA, USA

would benefit the elderly population. The residential care facilities (called établissements medico-sociaux) that care for individuals with serious disabilities in France, particularly those with severe forms of Autism Spectrum Disorder (ASD) and Intellectual Developmental Disorder (IDD) do not currently have such structured logistical support. Understandably, these facilities have proactively confined their residents since the early stages of the pandemic and applied stricter protective measures for them. However, the medicosocial establishments with residents infected with COVID19 requiring inpatient services have expressed their dismay at the meanders of a triage pathway that is poorly adapted to the care needs of people with ASD and IDD. The scientific literature is devoid, to date, on questions of screening and treatment of COVID-19 in people with ASD and IDD. How effective are the strategies used in the general population to protect them? How many are infected? What are the risk and protective factors and clinical expression of COVID-19 in this population? What has been the availability of intensive care services, including those requiring ventilatory support? The prevalence, clinical and prognostic impact of COVID19 in people with ASD and IDD therefore largely remain unknown.

It is apparent that as a highly vulnerable population, individuals with severe ASD and IDD will benefit from improved access to care (Lindly et al. 2019). They are exposed to similar conditions of vulnerability to COVID19 as the elderly. They live in community based housing with increased risk of viral transmission. They carry greater burden of underlying health conditions, particularly cardiovascular diseases (Miot et al. 2019), as well as possible premature aging (Hwang et al. 2020). They may have immune system alterations significantly associated with increased levels of inflammatory cytokines like C-reactive protein (CRP) and interleukin-6 (IL-6), that could translate into severe forms of COVID-19 as in the elderly population (Schoufour et al. 2016). Indeed, there is a specific serum 
inflammatory pattern with increased IL-6 correlated with ASD severity (Masi et al. 2017), thus potentially leading to a higher incidence of severe forms of COVID-19 among people with ASD, and IDD.

As many individuals with severe disabilities have difficulty communicating, they cannot express their symptoms early, making surveillance, prevention, and testing paramount in order for them to access proper care in the event of rapidly progressing infection, especially since many medical or resuscitation teams are unfamiliar with their specialized care needs. The physiological similarity to the elderly population may suggest comparable risks of atypical prodromal symptoms, complications, and mortality, in particular for aging people with ASD and IDD. These observations need to be considered in the context of aversion of people with ASD and IDD to physical, hematological, as well as radiological examinations, as they often trigger anxiety and adverse behavioral responses, especially when performed outside their familiar settings. Unavailability of virology diagnosis in these settings could also result in further diagnostic delays with ensuing increased morbidity and mortality. Furthermore, the younger persons with ASD and IDD in these residential care settings present alternate challenges: many may be asymptomatic carriers, increasing the risk of disseminating COVID-19 to other vulnerable groups, and healthcare workers, who are already at increased risk of contracting the disease due to the shortage of personal protective equipment (PPE) combined with extended work hours that make them at increased risk.

\section{Conclusion and Perspectives}

The COVID-19 pandemic has led to an unprecedented global challenge in the care of individuals with ASD and IDD. At a time when new waves of the pandemic are predicted, maintaining safety from increased transmission risk of COVID-19 in residential and specialized inpatient settings is of paramount importance. In lieu of dearth of international recommendations for protection of individuals with ASD and IDD requiring strong assistance in daily living, we draw attention to the following perspectives:

\section{Infection Prevention and Control}

- Care needs to be provided in the safest way possible without discrimination on the basis of disability;

- In view of asymptomatic COVID-19 forms, there is a need to strictly maintain social distancing measures, wear masks or cloth face coverings, and to compartmentalize COVID-19 free and COVID-19 safe areas, as well as to systematically plan to conduct RT-PCR testing before any admissions and transfers;
- Since ASD and IDD are complex neurodevelopmental conditions, there is a need for infection prevention and control approaches adapted to the cognitive, behavioral, and physical care needs of individuals and in implementing all barrier measures;

- The standard approaches to delivery of care need to be adjusted to incorporate telehealth and videoconferencing services in order to facilitate rapid access to primary care, and specialist providers, including those in mental and behavioral health, to access platforms with expertise in ASD and IDD, to address pressing COVID-19, as well as non-COVID-19 related conditions, and to make referrals as appropriate;

- For continuity and minimal disruption of care, direct service and ancillary personnel need to be monitored daily for any COVID-19 related symptoms and contact and advised to stay home according to occupational health policies;

- Since professional practice needs to be daily guided, it is important to continuously share the global experiences, and new evidence-based knowledge and scientific breakthroughs.

\section{Screening}

- It is essential to promote a well-coordinated and continuous screening strategy among residents and caregivers, with contact exposure risk assessment, as well shared decision-making involving families, guardians, and referral partners involved in care pathways;

- For rapid, highly responsive screening, the facilities need to be self-sufficient in performing nasopharyngeal COVID-19 testing with continuous availability of appropriately trained staff and provision of requisite transportable screening test kits;

- Since individuals with ASD and IDD have severe adaptive difficulties, screening needs to be offered on-site with benefit of personalized care and insight into their needs and any anticipated risks.

\section{Compliance with Ethical Standards}

Conflict of interest The authors declare that they have no conflict of interest.

\section{References}

Hwang, Y. I., Foley, K. R., \& Trollor, J. N. (2020). Aging well on the autism spectrum: An examination of the dominant model 
of successful aging. Journal of Autism and Developmental Disorders, 50(7), 2326-2335. https://doi.org/10.1007/s1080 3-018-3596-8.

Lindly, O. J., Zuckerman, K. E., \& Kuhlthau, K. A. (2019). Healthcare access and services use among US children with autism spectrum disorder. Autism, 23(6), 1419-1430.

Masi, A., Breen, E. J., Alvares, G. A., Glozier, N., Hickie, I. B., Hunt, A., et al. (2017). Cytokine levels and associations with symptom severity in male and female children with autism spectrum disorder. Molecular Autism, 8, 63. https://doi.org/10.1186/s1322 9-017-0176-2.

Miot, S., Akbaraly, T., Michelon, C., Couderc, S., Crepiat, S., Loubersac, J., et al. (2019). Comorbidity burden in adults with autism spectrum disorders and intellectual disabilities-A Report From the EFAAR (Frailty Assessment in Ageing Adults With Autism Spectrum and Intellectual Disabilities) Study. Front Psychiatry, 10, 617. https://doi.org/10.3389/fpsyt.2019.00617.

Schoufour, J. D., Echteld, M. A., Boonstra, A., Groothuismink, Z. M., \& Evenhuis, H. M. (2016). Biochemical measures and frailty in people with intellectual disabilities. Age and Ageing, 45(1), 142-148. https://doi.org/10.1093/ageing/afv152.

Publisher's Note Springer Nature remains neutral with regard to jurisdictional claims in published maps and institutional affiliations. 